\title{
LA COMUNIDAD ANDINA DE NACIONES Y LA EVOLUCIÓN DEL PROCESO DE INTEGRACION SOCIOECONÓMICO EN LATINOAMÉRICA ${ }^{1}$
}

\author{
Ernani Contipelli
}

doi: 10.18543/ed-64(1)-2016pp261-280

Sumario: 1. Introducción. 2. Breve AnÁlisis Histórico InStituCiOnal. 3. Estructura y Funciones de los Principales Órganos E Instituciones. 4. CATEgorización DEL Proceso de INTEGRACión ANDINO. 4.1. Evolución de las Propuestas de Integración. 5. CONClUSIÓN. 6. REFERENCIAS BIBLIOGRÁFICAS.

\section{INTRODUCCIÓN}

A finales de la década de 60, un grupo de países ubicados en la región andina compuesta por Bolivia, Colombia, Chile, Ecuador y Perú, decidió firmar un pacto por el que pretendían, a través de la integración y de la cooperación económica, superar las adversidades causadas por el subdesarrollo, empezando, así, el proceso de integración andino conocido como Pacto Andino o Acuerdo de Cartagena.

La evolución del Pacto Andino, en la actualidad, Comunidad Andina, se caracteriza por una serie de avances y retrocesos comunes a las dificultades

1 Trabajo realizado en el marco del Proyecto de Investigación Constitución y mercado en la crisis de la integración europea. $\mathrm{I}+\mathrm{D}+\mathrm{i}$ del Programa Estatal de Investigación, Desarrollo e Innovación orientada a los Retos de la Sociedad, del Ministerio de Economía y Competitividad (DER2013-48327-C3-1-R). Investigadores principales: José Luis GARCia Guerrero y María Luz Martínez Alarcón. 
encontradas en cualquier proceso de integración. Aunque la intención del Pacto Andino sea acercar los países andinos para promover el desarrollo armónico y equilibrado en la región por intermedio de acciones conjuntas, la crisis instaurada por los conflictos entre sus miembros pone en riesgo el futuro del modelo de integración.

El presente artículo tiene por objeto realizar una descripción general del proceso de integración andino a partir de su análisis histórico, institucional y conceptual. De ese modo, en la primera parte, presentamos un breve análisis histórico del desarrollo institucional de la integración andina, verificando los principales hechos que contribuirán para su formación y que actúan en su conformación actual. La segunda parte explora la estructura institucional de la Comunidad Andina para verificar la adecuación de sus órganos e instituciones con los objetivos estipulados para el bloque. En la tercera parte, tratamos la categorización del regionalismo andino y la evolución de sus propuestas institucionales, especialmente, para verificar como la Comunidad Andina se comporta ante el actual escenario globalizado, como vía para debatir el propio cuadro del desarrollo del proceso de integración socioeconómica en Latinoamérica.

\section{BREVE ANÁLISIS HISTÓRICO INSTITUCIONAL}

Con la desilusión generada por la ineficiencia de la ALALC (en adelante, Asociación Latinoamericana de Libre Comercio) como entidad integracionista destinada a propiciar desarrollo económico en el continente sudamericano, por la falta de consideración de las diferencias existentes entre sus miembros y de las constantes crisis políticas que imperan en el continente ${ }^{2}$, algunos países buscaron en la formación de distintos bloques que atendían a sus peculiaridades regionales la respuesta a sus problemas económicos para, posteriormente, pensar en la incorporación a un Mercado Común Latinoamericano, como, por ejemplo, en 1966, la Declaración de Bogotá «Bases de una

${ }^{2}$ Como señalado una de las grandes motivaciones para la firma del Acuerdo de Cartagena, además de la pretensa uniformidad económica en la región andina, esta relacionada con la insatisfacción de determinados países miembros de la ALALC en especial los subdesarrollados por la ausencia de mecanismos compensatorios. En tal sentido, GonZÁLES afirma que: «Los países medianos y pequeños que se encontraban abocados a formar sus propias estructuras continuaron desarrollando principalmente sus políticas de sustitución de importaciones para sus propios mercados, ya que su potencialidad para aprovechar el mercado ampliado requería una acumulación inicial de infraestructura que no poseían. Este elemento creó el descontento de los países medianos y pequeños que en 1969 promovieron el acuerdo sub-regional andino» (GONZÁLES, A. C. Reflexiones sobre el Futuro de la ALALC. Integración Latinoamericana: El Futuro de la ALALC, p. 14. Revista Mensual INTAL n. ${ }^{\circ}$ 40, 1979). 
Común Política Internacional» firmada por Chile, Ecuador, Perú y Venezuela. Con vistas a la formación de un mercado común entre esos países, que, al fin, se convirtió en una primera manifestación formal de integración entre los países andinos. Algunos autores, como LuNA CAMPO, consideran que la historia del Grupo Andino empieza a gestarse con la suscripción de la «Declaración de Bogotá» en 1966, en que fue posible establecer las bases preliminares del futuro pacto de integración subregional ${ }^{3}$.

Posteriormente, la integración andina se profundiza con la subscripción del Acuerdo de Cartagena en 26 de mayo de 1969 entre Bolivia, Colombia, Chile, Ecuador, Colombia y Perú, que efectivamente crea el Pacto Andino, configurando una unión aduanera entre los mencionados países. En sus bases originales, ese modelo de integración era definido como un esfuerzo para fortalecimiento del poder de negociación para mejor actuación en el comercio internacional y reducción de la dependencia. Sobre la composición del grupo, cabe resaltar que en enero de 1971, Venezuela notificó a la ALALC su intención de incorporarse al Acuerdo de Cartagena, lo que efectivamente ocurrió en febrero de 1973.

Por lo tanto, el Pacto Andino surge de la necesidad de preparar la economía de los países de la región para las exigencias del mercado internacional, estimulando el comercio interregional, la industrialización y el consecuente armónico desarrollo de sus miembros. En ese sentido, al menos teóricamente, la integración impulsaría el acceso recíproco a los mercados de los países miembros del grupo, dotándoles de mayor poder de negociación para inserción en el plan internacional, la medida en que permitiría una actuación con unidad y una base para tratar igualmente a todos los países del continente. Constituía un esfuerzo conjunto para superar la condición de subdesarrollo,

${ }^{3}$ Luna CAmpo, A. Elementos de Derecho Comunitario: Panorámica de la ALALC y del Pacto Andino, p. 41 Bogotá: PAX, 1974. La Declaración de Bogotá, firmada en 16 de agosto de 1966, originada de una reunión envolviendo los Presidentes de Colombia, Chile y Venezuela y los delegados personales de los Presidentes de Ecuador y Perú, en que se pretendía discutir la situación económica-social de eses países en el contexto Latinoamericano, evoluciono en dirección a la suscripción del mencionado documento tendente fundamentalmente a la creación de un mercado común subregional, que según Leopoldo Duran Valdés, «aparte de reconocer la urgencia de la integración de las naciones latinoamericanas en una gran comunidad económica señala, en forma específica, la necesidad de acelerar al máximo el progreso de los países de menor desarrollo económico y de mercado insuficiente, para lo cual propone, entre otras medidas, 'la concertación de acuerdos entre uno o más países de menor desarrollo económico relativo y de mercado insuficiente con un país de mayor desarrollo económico relativo, que estarían libremente abiertos a la adhesión de los países pertenecientes a las dos categorías primeramente mencionadas pero cuyas ventajas temporalmente no serian extensiva a las restantes» (El Acuerdo de Integración Subregional: Antecedentes, Negociaciones y Comentarios, p. 179. Revista de Derecho Publico n. ${ }^{\circ}$ 10. Santiago: Universidad de Chile, 1969). 
especialmente, por la reducida dimensión de sus mercados internos que no despertaban intereses del comercio internacional.

La puesta en marcha del proceso de integración exigió la flexibilización de las medidas adoptadas para concilian de los intereses de los países involucrados y alcance de las finalidades propuestas por el Pacto Andino, como, por ejemplo, la reprogramación de plazos para liberación del comercio interno en la subregión acordado a través del Protocolo de Lima (1976). Sin embargo, el proceso integracionista andino sufre un duro golpe en 30 de octubre 1976, con la retirada de Chile (Decisión 102: «Cese para Chile de los derechos y obligaciones derivados del Acuerdo de Cartagena», justificada en divergencias sobre las medidas económicas, en especial, relativas al libre comercio adoptadas por el grupo. En realidad, recordamos que durante tal momento el país pasaba por un periodo político turbulento con la reciente derribada de Salvador Allende del poder y el golpe militar comandado por Augusto Pinochet ${ }^{4}$.

Otra situación que afectó el avance de la propuestas del Pacto Andino fue la crisis económica de 1980, la cual llevó a los países miembros a promover políticas proteccionistas, reduciendo las posibilidades de lograr los objetivos acordados, sobre todo, en relación a la unión aduanera, elemento fundamental para definición de políticas comunes y aumento de la competitividad del bloque ante el comercio internacional. En tal contexto, fue firmado el Protocolo de Quito (1987), que flexibilizaba los procedimientos definidos por Acuerdo de Cartagena y acababa por postergar el desarrollo del proceso de integración. Así, durante tal período la restricción al crecimiento generada por crisis económica, asociada a una restricción al crecimiento, influenció negativamente en los flujos comerciales entre los países miembros del bloque, implicando fuertes trabas al desarrollo del proceso de integración andino $0^{5}$.

Durante finales de los ochenta e inicios de los noventa, fueron realizados diversos arreglos institucionales y procedimentales con vistas a conferir mayor viabilidad al Pacto Andino ${ }^{6}$, especialmente, a través de la creación de una

${ }^{4}$ Con respecto a la retirada de Chile del Pacto Andino, CASAs Gragea afirma que: «Bajo el régimen del general Pinochet, Chile adoptó un modelo económico liberal de orientación hacia fuera que el resto de países del Acuerdo consideraron incompatibles con la estrategia y los objetivos conjuntos establecidos. Finalmente, esto supuso el abandono de Chile del Grupo Andino en 1976 y como consecuencia, un duro retroceso en el proceso comunitario andino» (El Modelo Regional Andino Enfoque de Economía Política Internacional, p. 16. Quito: Universidad Andina Simón Bolívar, 2003).

5 Porta, F. La Integración Sudamericana en Perspectiva. Problemas y Dilemas, p. 26. Santiago: CEPAL, 2008.

${ }^{6}$ Durante la década de los 90 s, constatamos un fortalecimiento del proceso de integración andino vinculado a los siguientes factores: en el ámbito domestico, por la volun- 
zona de libre comercio y institución del arancel externo común entre el bloque, destacándose las decisiones tomadas en la Cumbre de Galápagos (1989), donde fue formulado el «Diseño Estratégico para la Orientación del Grupo Andino"7; en la Reunión de Machu Picchu (1990), que determina la constitución del Consejo Presidencial Andino; en la Reunión de Caracas (1991), la cual ratificó los acuerdos para institución del arancel externo común y determino la armonización de políticas cambiarias y monetarias; y, finalmente, la Reunión de Cartagena de Indias (1991), fundamental en la definición de las políticas externas del grupo, que culmino en importantes tratativas comerciales como con los países miembros del MERCOSUR.

Con respecto al arancel externo común, en 1992, Bolivia decidió el levante de las tasas arancelarias en relación a las importaciones de Colombia, Ecuador y Venezuela; en 1993, Ecuador programó la suspensión de las tarifas arancelarias para los países miembros del Pacto Andino y, finalmente, en el mismo año, el Arancel Externo Común fue aprobado a través de la Decisión 335 de la Comisión del Acuerdo de Cartagena.

En 1997, el bloque experimenta un giro institucional y estructural por intermedio de los Protocolos de Trujillo (1996) y Sucre (1997), con la introducción de reformas en las bases originales del Acuerdo de Cartagena, las cuales amplían el campo de actuación del Pacto Andino. De ese modo, la integración pasa a ser no apenas técnica y comercial, sino también de contenido claramente político, al insertar en su estructura el Consejo Presidencial Andino y el Consejo Andino de Ministros de RR.EE., además de convertir Junta del Acuerdo de Cartagena en un Secretaria General con funciones políticas, originando, así, el Sistema Andino de Integración y la Comunidad Andina en reemplazo del Pacto Andino.

Pese el aumento de los indicadores económicos y comerciales con la nueva configuración institucional del grupo, los niveles de desigualdad social y pobreza no presentaban mejoras, llevando a la extensión de los objetivos

tad política de apertura de los mercados de los países miembros del bloque, influenciados por el Consenso de Washington; y, en el plan internacional, por la intensificación del comercio mundial e internacionalización del capital. Tal fase del Pacto Andino es caracterizada por el denominado regionalismo abierto, que busca conciliar la interdependencia y liberalización comercial, orientando el proceso de integración a adopción de políticas tendentes al incremento de la competitividad internacional.

7 Especialmente, en la Cumbre de Galápagos, en que fue aprobado el Diseño Estratégico y el Plan de Trabajo, constatase una inversión en el modelo de sustitución de importaciones que vigoraba en las ideas iniciales del grupo, las cuales tendían a la protección de la industria nacional con elevados aranceles, pasando al modelo abierto, a partir de los finales de la década de ochenta, con la eliminación de los aranceles internet y formación de una zona de libre comercio, ocurriendo un aumento considerable en el comercio intracomunitario. 
buscados por la integración andina con la incorporación de la vertiente social, conforme decisión tomada en la Cumbre de Quirama del Consejo Presidencial Andino (2003), sobre todo, con la creación del Consejo Andino de Ministros de Desarrollo Social y la aprobación del Plan Integrado de Desarrollo Social - PIDS.

A partir del nuevo arreglo institucional por la creación de la Comunidad Andina y del Sistema Andino de Integración y también por la mayor complejidad y ambición de los retos definidos para el bloque, en 2005, fue insertado en su agenda entre los objetivos primordiales: la promoción de la integración latinoamericana, con la intención de estimular su actuación política en el plan internacional ${ }^{8}$, transformando la Comunidad Andina en un canal de articulación entre Sudamérica, Centroamérica y el Caribe. En ese mismo año, Brasil, Argentina, Paraguay y Uruguay, países componentes del MERCOSUR fueron convertidos en miembros asociados de la Comunidad Andina (Decisión 613 del Consejo Andino).

Por otro lado, en 2006, después de algunos miembros del bloque anunciasen el establecimiento de acuerdos de libre comercio con Estados Unidos, Venezuela opta por su salida de la Comunidad Andina, mientras que, en el mismo año, el Consejo Andino de Ministros de Relaciones Exteriores aprueba la concesión a Chile de la condición de País Miembros Asociado, posibilitando un acercamiento con vistas al reingreso del país al bloque ${ }^{9}$.

${ }^{8}$ Entre las importantes tratativas realizadas por la Comunidad Andina para promover su inserción internacional y establecer pactos sobre la base «región a región», señalamos las negociaciones, tras el Cumbre de Viena en 2006, para firma del Acuerdo de Asociación Unión Europea-CAN, lo cual objetiva el fortalecimiento del proceso de integración en la región, diferenciándose del foco comercial presente en otros acuerdos negociados anteriormente de forma bilateral por los estados miembros de la CAN (LEVí CoRAL, M. La Relación UE-Comunidad Andina en el Contexto de la Negociación de los Acuerdos de Asociación, p. 252, en Roy, J. y Domínguez, R. (eds). España en la Unión Europea y el Estado de la Integración Latinoamericana. Miami: Miami-Florida European Union Center/Jean Monnet Chair, 2010), pasando a involucrar temas de contenido social y medioambiental, decisivos para formación de la posterior Estratégia Andina de Cohesión Económica y Social.

9 A pesar de la necesidad de formulación de un nuevo diseño estratégico para desarrollo del Pacto Andino con carácter multidimensional (economía, política y sociedad), tomando en consideración la transformaciones sufridas en el escenario internacional, «el inicio de negociaciones bilaterales por parte de Ecuador, Perú y Colombia para concretar tratados de libre comercio con Estados Unidos, y la salida de Venezuela de la CAN en abril de 2006, empantanaron los avances realizados (...) y plantearon un nuevo panorama de crisis, al punto que diversos sectores le han venido apostando a la desintegración del grupo» (CASAs Casas, A. y Correa, M. E. ¿Qué Pasa con la Comunidad Andina de Naciones-CAN? Papel Político vol. 12, n. ${ }^{\circ}$ 02. Bogotá: Pontificia Universidad Javeriana, 2007). Entretanto, comprendemos que el bloque resiste y presenta un señal de fortalecimiento 
La retirada de Venezuela no afecta la ampliación de los objetivos propugnados por el bloque, que, en la Cumbre de Tarija (2007), acuerda un modelo de Integración Integral para atribuir a la aspectos políticos y sociales, ambientales y culturales, la misma preocupación conferida a la agenda económica y comercial del grupo ${ }^{10}$.

Esa integración integral es reforzada por la definición de Agenda Estratégica Andina y su consecuente plan de implementación (2010), así como por la elaboración de una Estrategia de Cohesión Económica y Social ${ }^{11}$ y de los Objetivos Andinos de Desarrollo Económico y Social, que fijaran ámbitos de cooperación, compromisos y metas concretas que deberán ser asumidas por el grupo hasta el 2019, para formular políticas publicas comunes destinadas a fomentar el desarrollo social conjunto, actuando en la reducción de la pobreza y de las desigualdades sociales.

\section{ESTRUCTURA Y FUNCIONES DE LOS PRINCIPALES ÓRGANOS E INSTITUCIONES}

Desde el Acuerdo de Cartagena, el proceso de integración andina surge con una estructura institucional caracterizada por la supranacionalidad, presentando inicialmente como principales Órganos la Comisión del Acuerdo de Cartagena, el Tribunal Andino de Justicia ${ }^{12}$, el Parlamento Andino y la

durante tal periodo, especialmente, por el reingreso de Chile como Estado miembro asociado, que le permite participar de reuniones del SAI (artículo 2 de la Decisión 645).

${ }^{10} \mathrm{La}$ Integración Integral convirtiese en el nuevo paradigma de las acciones de la Comunidad Andina, tratándose de actualización de sus objetivos a las exigencias del multipolar contexto internacional contemporáneo que se presenta marcado por distintas y dinámicas formas de desarrollo institucionales y actores, en especial referencia al desarrollo sostenible para atribuir condiciones dignas de existencia a población de esos países, conforme mencionado por los Jefes de Estados de los miembros de la Comunidad en la Cumbre de Tarija sobre la necesidad de «desarrollar y profundizar el proceso de integración de la Comunidad Andina tomando en cuenta, en forma más efectiva, las visiones y enfoques de los Países Miembros, con el objetivo de lograr una unidad en la diversidad al servicio del vivir bien de nuestros pueblo y en armonía con la naturaleza» (2007).

${ }^{11}$ La Estrategia Andina de Cohesión Económica y Social fue elaborado en el marco del proyecto de cooperación CAN-Unión Europea de Apoyo a la Cohesión Económica y Social (CESCAN) a partir de la estrategias de cooperación regional 2007-2013, tratándose de la adopción de políticas sociales comunes y prioritarias direccionadas a «contribuir a la superación de la pobreza, la exclusión y desigualdad social en el área andina; a la vigencia de los derechos sociales, individuales y colectivos de la población $\mathrm{y}$ al logro de un mayor grado de cohesión nacional y regional» (Convenio n. ${ }^{\circ}$ DCIALA/2007/018-993, 2008).

12 El Tribunal Andino tiene entre sus funciones primarias la tarea de control de la legalidad de las normas comunitarias, a través de la acción de nulidad, la resolución de las 
Junta del Acuerdo de Cartagena (JUNAC). A partir del Protocolo de Quito, adquiere importancia el Consejo Presidencial Andino y el Consejo de Ministros de Relaciones Exteriores.

La conformación de la estructura actual del Pacto Andino define sus estructuras institucionales en el Protocolo de Trujillo (1997) ${ }^{13}$, donde la responsabilidad por el desarrollo de proceso de integración no se encuentra a cargo de la Comisión, instancia de carácter esencialmente, comercial, sino es que es determinado por el Consejo Presidencial Andino y al Consejo Andino de Ministros de Relaciones Exteriores, Órganos dotados de poder político. Lo que demuestra la nítida tendencia de ampliar los objetivos de bloque para asumir una orientación de carácter político y no apenas de contenido económico-comercial.

El Consejo Presidencial Andino está compuesto por los Presidentes de Bolivia, Colombia, Ecuador y Perú, siendo considerando el máximo Órgano del bloque, ejerce las funciones de conducción política de la Comunidad Andina, formalizadas en Directrices que no son consideradas parte integrante del orden jurídico comunitario. Por otro lado, compete al Consejo Andino de Ministros de Relaciones Exteriores la tarea de elaboración de la política exterior de los Estados-miembros relativa a la integración, además, de ser necesario, la coordinación de posiciones comunes ante instancias internacionales. Por último, la Comisión tiene la función de formular, ejecutar y evaluar la política de integración en que se refiere a la temática comercial y de inversiones, formulando normas que deben ser cumplidas por los países de la Comunidad.

diferencias sobre el incumplimiento de las obligaciones entre los países miembros y asegurar la interpretación y aplicación uniforme de la legislación comunitaria en el territorio de la Comunidad Andina. Además de las acciones a disposición de los Estados, es posible verificar algunas posibilidades de provocación del Tribunal por particulares, como por ejemplo, la acción de nulidad de los actos normativos comunitarios, lo que revela el intento de acerca la ciudadanía del proceso de integración. El Tribunal Andino también posee competencias de carácter arbitral y de jurisdicción laboral con respecto a los funcionarios comunitarios (Pino Estrada, M. M. El Sistema Juridico en la Comunidad Andina de Naciones. Revista Intellector n. ${ }^{\circ}$ 04, Rio de Janeiro: Centro de Estudos em Geopolitica e Relacoes Internacionais, 2006). Importante señalar que el Acuerdo de Cartagena no había previsto originalmente la creación de un tribunal, atribuyendo la resolución de controversias al sistema de arbitraje. En 1979, a través del Tratado de Creación del Tribunal de Justicia de la Comunidad Andina, que paso a tener vigencia apenas en 1983, estableció la institucionalización de un sistema judicial comunitario en el marco del Pacto Andino.

13 De acuerdo con Marcel TANGARIFE, la importancia decisiva del Protocolo de Trujillo consiste en el hecho de que tal documento trae «cambios para el proceso de integración, especialmente en lo referente al fortalecimiento de las instituciones comunitarias, para dotar a la Comunidad Andina de mayores instrumentos encaminados a perfeccionar la zona de libre comercio y la unión aduanera» (Derecho de la Integración en la Comunidad Andina, p. 63. Bogotá: Baker \& McKenzie, 2002). 
El Consejo Andino de Ministros de Relaciones Exteriores y la Comisión pueden ser considerados como los órganos legislativos en la estructura del bloque, ya que son encargados de expedir las denominadas Decisiones, o leyes andinas. Conforme al Acuerdo de Cartagena, la producción legislativa de tales órganos obliga a los países miembros ${ }^{14} \mathrm{y}$ posee aplicabilidad inmediata $^{15}$, siendo de fundamental importancia para consolidar la actuación de la Comunidad bajo las determinaciones del derecho comunitario andino.

La articulación entre órganos e instituciones que poseen diferentes competencias y funciones -normativas, políticas, judiciales, ejecutivas, entre otrasatribuye a la Comunidad Andina una estructura y complejidad similar a un «Estado», que está a cargo del Sistema Andino de Integración (SAI). Por lo tanto, el Sistema Andino de Integración tiene como objetivo la coordinación entre las instancias componentes de la Comunidad Andina para profundizar el proceso de integración y fortalecer las acciones conjuntas entre sus miembros.

Entre los mecanismos de coordinación y funcionamiento del SAI, encontrase la Reunión de Representantes de la Instituciones componentes del Sistema, que es convocada y dirigida ordinariamente, al menos una vez al año, por el Presidente del Consejo Andino de Ministros de Relaciones Exteriores y, extraordinariamente, cuando solicitad por cualquiera de las instituciones integrantes. El SAI es responsable también por la articulación de los Consejos Asesores Ministeriales que expresan opiniones al Consejo Andino de Ministros de Relaciones Exteriores y de los Comités Técnicos establecidas en el marco del proceso de integración.

La Secretaria General del SAI acumula funciones de apoyo técnico, correspondientes anteriormente a la antigua Junta del Acuerdo de Cartagena, y también ejecutivas a través de la emisión de Resoluciones. Otra instancia de carácter comunitaria es el Parlamento Andino que, sin potestad para expedición de normas vinculantes, en la medida en que colabora con la creación de normas mediante sugerencias a los órganos del Sistema Andino de Integración y actúa en la verificación del cumplimiento de los objetivos del proceso de integración. Funciona como un simple órgano deliberativo que es susceptible de diversas críticas, ya que al no propiciar una efectiva representación democrática de la ciudadanía ante el proceso de integración y carecer de me-

${ }^{14}$ El articulo 2 del Acuerdo de Cartagena estipula que: «Las Decisiones obligan a los países Miembros desde la fecha en que sean aprobadas por el Consejo Andino de Ministros de Relaciones Exteriores o por la Comisión de la Comunidad Andina».

${ }^{15}$ El articulo 3 del Acuerdo de Cartagena determina que las Decisiones del Consejo del Consejo Andino de Ministros de Relaciones Exteriores o de la Comisión, así como de las Resoluciones de la Secretaría General «serán directamente aplicables en los países Miembros a partir de la fecha de su publicación en la Gaceta Oficial del Acuerdo, a menos que las mismas señalen una fecha posterior». 
canismos que posibiliten una efectiva canalización de las propuestas sociales objetivadas por la Comunidad, se pondría en cuestión su propia existencia.

La coordinación empeñada por el SAI se ha fortalecido a partir de la adopción del paradigma de Integración Integral y la consecuente definición de los Objetivos Andinos de Desarrollo Social, que requiere una mayor participación política del Consejo Andino de Ministros de Desarrollo Social y de sus respectivas instancias nacionales para estrechamiento de los vínculos de cooperación interinstitucional.

Desde un punto de vista práctico, el gran problema del Sistema de Integración Andino respecto a la convergencia y cumplimiento de acciones basadas en el Derecho Andino, está relacionado con el principio de unidad que «impone y permite garantizar un funcionamiento institucional coherente y centrifugo, en el que la actuación de los distintos órganos se oriente en una misma dirección, evitando la dispersión institucional» ${ }^{16}$.

Sobre tal aspecto, el Sistema Andino de Integración revela cierta ineficacia por la ausencia de coordinación entre sus Órganos e Instituciones, lo que puede ser constatado en los siguientes aspectos: «i) No acatamiento de los fallos de los órganos del Sistema Andino de Integración, por la prioridad asignada a las consideraciones locales o internas de los países sobre los intereses comunitarios; ii) Por la énfasis dado en la estructura institucional de la Comunidad Andina a las actividades adelantadas por entes ejecutivos como los Ministerios de Relaciones Exteriores, la Comisión y la Secretaría General de la Comunidad Andina, iii) Por la inexistencia de políticas de coordinación de los órganos e instituciones del Sistema Andino de Integración en el marco del Derecho Andino de la Integración ${ }^{17}$.

Por fin, aunque la Comunidad Andina presente una estructura institucional compleja y establece en sus propuestas el cometido de lograr de una integración de carácter supranacional, su realidad es muy diferente. La dinámica de sus instituciones revela un proceso de integración de carácter intergubernamental en que los intereses nacionales de los países miembros dominan el direccionamiento político de la Comunidad, debilitando el carácter comunitario de sus instancias decisionales, siendo por lo tanto, cuestionable la estructura existente con la realidad actual del bloque ${ }^{18}$.

${ }^{16}$ QuINDIMIL, J. Instituciones y Derecho de la Comunidad Andina, p. 145/146. Bogotá: Tirant lo Blanch, 2006.

17 Blanco Alvarado, Carolina. La Crisis de la Comunidad Andina. Revista de Derecho, Universidad del Norte, 42: 1-32, Barranquilla, 2014.

${ }_{18}$ Podemos ilustrar la situación con la Decisión 322 que permitió a los países miembros de Comunidad a promover negociaciones unilateralmente con otros países de la región (ALADI, Centroamérica y Caribe), estando apenas obligados a comunicar tal hecho a los demás miembros de la Comunidad, contrariando el articulo 98 del Acuerdo de Car- 
De todas maneras, señalamos que la Comunidad Andina de Naciones está lejos de ser vista un ente supranacional, con personalidad jurídica internacional soberana que se sobreponga a los países miembros. En tal sentido, el proceso de integración no se desarrolla a través de la transferencia de soberanía con carácter definitivo, sino con la atribución de competencias respetando el orden jurídico constitucional de los países miembros.

Dentro de la perspectiva de derecho comunitario y tomando en consideración la estructura institucional de la Comunidad Andina, constatamos que su proceso de integración sigue una concepción propia, sui generis, en la medida en que los poderes de los países miembros pueden ser limitados por las leyes comunitarias que prevalecen sobre la legislaciones estatales. Estas normas pueden ser aplicadas por el Poder Judiciario nacional, pero eso no implica en la pérdida de soberanía estatal, especialmente, en términos de legitimidad. Sin embargo, la inoperatividad de algunas instituciones claves perjudica la consolidación y desarrollo del Derecho Comunitario, como son los casos, por ejemplo, del Tribunal Andino, pues, aunque sus fallos sean vinculantes y de estricto cumplimiento, sus determinaciones no son cumplidas, implicando en un vacío de credibilidad en la actuación de tal entidad. Además, el Parlamento Andino, posee atribuciones deliberativas y de verificación sobre el desarrollo del proceso de integración, que no tienen carácter vinculante, reduciendo la importancia de su campo de acción ${ }^{19}$.

\section{CATEGORIZACIÓN DEL PROCESO DE INTEGRACIÓN ANDINO}

En las concepciones de regionalismo, el aspecto geográfico, por fuerza del fenómeno natural de la proximidad territorial entre países, funciona como un elemento facilitador para el establecimiento de relaciones políticas convergentes que pueden variar desde cuestiones económicas o socioculturales, entre otras, dentro de una zona espacial de interés común.

No obstante, debemos considerar que el hecho de estar ubicado en un mismo espacio geográfico y poseer rasgos generales comunes no significa que un trabajo conjunto desempeñado por las naciones comprendidas en la región será exitoso. Especialmente, en términos de integración, se evidencia la necesidad de establecimiento de arreglos institucionales y procesos que enganchen los participantes en un sentimiento de solidaridad y pertenencia.

\footnotetext{
tagena, que exige la realización de consultas a la Comisión «adquirir compromisos de carácter arancelario con países ajenos a la Subregión», lo que evidentemente prioriza los intereses nacionales en detrimento de los comunitarios, reforzando el carácter intergubernamental asumido por el bloque.

19 Casas Casas, A. y Correa, M. E. ¿Qué Pasa con la Comunidad Andina de Naciones-CAN? Papel Político vol. 12, n. ${ }^{\circ}$ 02. Bogotá: Pontificia Universidad Javeriana, 2007.
} 
Particularmente, en lo que respecta a la región andina, encontramos la cadena de montañas de los Andes como aspecto geográfico caracterizador de la zona y que, de cierto modo, refleja en la cultura del pueblo atribuyéndoles un rasgo común. Debemos considerar también los desafíos ocasionados por las barreras naturales de la región, como, por ejemplo, los desniveles de altura, que agregan factores de complejidad a la aproximación entre los países de la región, exigiendo un mayor esfuerzo en la superación de adversidades físicas para promoción de la integración en la zona.

Entretanto, es obvio que tal condicionante geográfico impone un acercamiento inicial que depende esencialmente de las voluntades políticas e intereses de los gobernantes de establecer vínculos comunitarios entre países ubicados en la región ${ }^{20}$. Por lo tanto, los aspectos geográficos y políticos se presentan como uno de los principales elementos en la determinación de los procesos de integración, como en el caso de la Comunidad Andina de Naciones, en que desde la firma del Acuerdo de Cartagena es posible constatar periodos de mayor avances, así como de retrocesos, siempre pendientes de los intereses nacionales de los países miembros.

Destacamos también que la existencia de una gran diversidad interna económica, en que encontramos países en creciente desarrollo como Perú y Colombia, agravan las diferencias políticas e ideológicas de los actuales gobiernos, generando un fraccionamiento al interior del bloque. Al fin, la coyuntura política genera efectos prejudiciales en la integración de la Comunidad Andina, limitando el establecimiento de elementos primordiales en la región como cohesión económica y social, que afecta negativamente al proceso de integración e incrementa la desigualdad entre los países miembros, como será comprobado en la secuencia de nuestro estudio.

\subsection{Evolución de las Propuestas de Integración}

Como forma de iniciar el presente apartado, importa considerar que los procesos de integración ${ }^{21}$ presentan determinadas etapas vinculadas a su pro-

${ }^{20}$ Con respecto a la complejidad de los factores que concurren para formación de las entidades regionales, MAZZEI afirma que: «Perché si sviluppi il regionalismo tra gli Stati non bastano la prossimità geografica e un certo grado di interdipendenza economica, ma sono necessari anche altri fattori quali l'identità regionale (per cui un insieme di tradizione storiche, culturali e sociali porta alla percezione condivisa di appartenere a una particolare comunità, o preferenze politiche o ideologiche. Il diverso peso specifico di questi fattori produce diversi tipi di regionalismo» (Relazioni Internazionali, p. 151. Milano: Egea, 2012).

${ }^{21}$ Para mejor comprender el concepto de integración es necesario ingresar en su sentido etimológico consistente en el acto de agregar diferentes especies, la unión de algo menor con algo mayor, de tal manera que el resultado de esa junción siempre implica en una variación positiva, de contenido superior a las condiciones anteriores. En el plan de la 
fundización y consolidación que empieza, en su periodo inicial, con la institución de una zona de libre comercio, en que son suprimidos los aranceles al comercio interregional. Secuencialmente, se establece la Unión Aduanera, con la uniformización de los aranceles en relación a las mercancías importadas de terceros países. Después se forma un Mercado Común con el objetivo de liberar el tránsito de los factores productivos en la zona territorial correspondiente al bloque ${ }^{22} ; \mathrm{y}$, por último, se establecen políticas macroeconómicas unificadas para establecer la Unión Económica entre los países miembros ${ }^{23}$.

ciencia política, comprende la unión de Estados soberanos para constitución de una comunidad con existencia y actuación internacional propia. Específicamente, la integración regional se refiere al proceso en que diferentes Estados soberanos, situados en una misma zona geográfica del planeta se unen para formación de una organización internacional (CONTIPELli, E. y PicCiAu, S. La Evolución de los Modelos de Cooperación Internacional y las Potencias Emergentes: BRICS, p. 31, Revista Direito Mackenzie v. 08, 2014).

${ }^{22}$ La formación del Mercado Común Andino (MCA) tuvo sus rumbos definidos especialmente por la Cumbre Presidencial de Lima (2000), en que los Jefes de Estado de los países miembros decidieran, justificados en la atribución de agilidad y flexibilidad al proceso, promover el desarrollo del mercado común por intermedio de Directrices, la cuales no hacen parte del orden jurídico comunitario y, en tal perspectiva, manifiestan las voluntades políticas individuales y puntuales de cada Estado, es decir, demuestra directamente el interés de los mandatos presidenciales, perjudicando el establecimiento de un programa consistente y de largo plazo, además de la ausencia de confianza en el funcionamiento institucional del proceso de integración.

${ }^{23}$ Según García Guerrero, en la ciencia económica constatamos cuatro tipos de acuerdos supraestatales que se encuentran directamente vinculados con la intensidad de su integración económica: acuerdos de libre comercio, caracterizados por la reducción, cuando no eliminación, de las barreras arancelarias a los productos comerciales, surtiendo efectos en el principio de unidad económica nacional y su correlato la unidad de mercado; uniones aduaneras, en que se profundiza la integración, pues, además de las barreras arancelarias, son fijados objetivos comunes frente al exterior, es decir se busca una política comercial común; mercado común, que implica una política comercial común y una completa liberalización de los factores de producción, generando un nuevo ordenamiento supraestatal que se relaciona con los ordenamientos de los estado miembros principalmente a través del principio de competencia; y el cuarto e ultimo tipo, corresponde a la unidad económica supra estatal, que conlleva las mismas exigencias que el mercado común, pero incluye, además, una armonización de las políticas macroeconómicas y de las políticas regulatorias. De acuerdo con el autor, el Pacto Andino estaría inserto en el segundo tipo de integración, o sea, unión aduanera, destacando que ese proceso de integración estaba dirigido a la conformación de un mercado común, pero ha fracasado, sobretodo, tras la creación de la Alianza del Pacifico involucrando México, Colombia, Perú y Chile. (GARCÍA-Guerrero, J. L. Las Integraciones Económicas Supraestatales y los Acuerdos entre Bloques Económicos en Definitiva, la Globalización como Último embate al Concepto Racional Normativo de Constitución, en Prats, E. J. y Jiminián, M. V. (coords) Constitución, Justicia Constitucional y Derecho Procesal Constitucional. Republica Dominicana: Instituto Dominicano de Derecho Constitucional, 2014). 
La superación de las etapas descriptas, que atribuyen complejidad al proceso de integración, encuentra una serie de factores que contribuyen para su limitación en el continente latinoamericano, entre los cuales se sobresalen la inestabilidad política y económica, las diferentes concepciones ideológicas que generan distintas perspectivas sobre la integración y la ausencia de apertura democrática en ciertos países.

Según la doctrina ${ }^{24}$, es posible identificar algunos aspectos que singularizan la formación del proceso de integración andino, relacionados con la determinación de una política de desarrollo industrial conjunta y ecuánime; la distribución uniforme de ventajas y costes de la integración, ese fue un motivos determinantes para formación del bloque contrariando la lógica de la ALALC; el establecimiento de un planteamiento estructurado, compuesto por etapas para lograr un arancel externo común; posibilitar la atracción de empresas multinacionales, sobre todo como forma de transferencia de tecnología; y, por fin, definición de estructura institucional capaz de generar potencializar los resultados positivos de la integración.

Teniendo en cuenta las afirmaciones anteriores revisaremos la evolución de las propuestas de integración del Pacto Andino. Es importante considerar algunas condicionantes que contribuyeran para creación del bloque y que están vinculados a aspectos socioculturales como el facilitador comunicacional en razón de la presencia de un idioma único en la región y los rasgos culturales comunes entre esos países miembros que se ubican en una misma región territorial.

De ese modo, el Pacto Andino se estableció como un modelo institucional destinado a la promoción de desarrollo económico en la región a través de la adopción de una política común para reducción de la dependencia directa en relación a la inversión extranjera, especialmente, con la armonización de normas relativas a la inversión extranjera en la región, diferenciándose de los demás esquemas de integración propuestos para países subdesarrollados. La protección a los países miembros del grupo revelan como puntos de acción la gradual eliminación de barreras aduaneras y la intensificación de las comunicaciones para facilitar el comercio recíproco.

Lógicamente, para alcanzar tales resultados el Pacto Andino estuvo cerca de 22 años entre progreses y retrocesos. Solamente, en 1989, la Cumbre de Cartagena determinó el renacimiento del proceso de integración andino, cuando los Presidentes de los países miembros acordaron la creación en un corto plazo de un modelo de Unión Aduanera, que fue dibujada con mayor precisión en las reuniones siguientes (Galápagos, Cuzco, La Paz, Caracas y

${ }^{24}$ Germánico, S. P. El Grupo Andino de Hoy: Eslabón Hacia la Integración de Sudamérica. Quito: Universidad Andina Simón Bolívar, 1995. 
Cartagena), las cuales decidirán por el establecimiento de la Zona de Libre Comercio y del Arancel Externo Común.

Por lo tanto, como principales resultados alcanzados a partir de las propuestas iniciales de formación del bloque, la Comunidad Andina logró establecer un modelo de integración comercial marcado por la existencia de una zona de libre comercio desde 1993, en que los productos de origen andino circulan libremente en la subregión (Bolivia, Colombia, Ecuador y Perú), independientemente del pago de aranceles, y de un arancel externo común en vigor desde 1995.

Otra importante conquista en términos de integración es la libre circulación de personas en el territorio de los países de la Comunidad Andina. A partir de 2003, fue autorizado el ingreso sin pasaporte entre los ciudadanos de los países miembros del bloque, bastando para entrada la presentación del respectivo documento de identidad. Además, con la Decisión 504 de Junio de 2001, fue creado el Pasaporte Andino, lo cual presenta un padrón uniforme con características mínimas en relación a la nomenclatura y a determinadas cuestiones de seguridad.

Con la adopción de la Agenda Estratégica Andina surgen nuevos retos, que necesitan de una mejor definición para consolidación de la integración andino, los cuales se refieren, por ejemplo, al desarrollo de la zona fronteriza en la región que posee un impacto negativo en el proceso, al generar tensiones entre los gobiernos de los países miembros, como el histórico conflicto sobre la salida al mar de Bolivia en que también están involucrado Chile y Perú, o el problema de los grupos criminales de narcotráfico y de los armados insurgentes, lo que exige una estrategia de cooperación uniforme entre los países andinos para actuar internamente en nivel local y regional en tales zonas. En la temática social, la Declaración de Machu Picchu destaca elementos clave que deben ser objeto de políticas públicas comunes entre los miembros de la Comunidad Andina como el refuerzo a la participación democrática, la defensa de los derechos de los pueblos indígenas y la mejora de las condiciones de vida de la población.

Aunque la Comunidad Andina haya logrado relevantes éxitos, las propuestas del ambicioso proyecto de integración no se concretaran en toda su dimensión, sobre todo, por la ausencia de apoyo político y de concordancia entre los miembros para el establecimiento de una política común. Es decir, la falta de una percepción única entre los gobernantes de los países miembros de la Comunidad Andina sobre el futuro del proceso de integración pone en riesgo su evolución.

Uno de los objetivos propuestos para el bloque y de fundamental importancia en su evolución, es la adopción de una política exterior común que está lejos de ser implementado, considerando las diferentes y contradictorias perspectivas ideológicas de los gobernantes de los países miembros. Por 
ejemplo, las negociaciones para el establecimiento de Tratados de Libre Comercio con Estados Unidos confrontan nítidamente las posiciones políticas de Colombia y Perú, con las de Bolivia y Ecuador, siendo incluso ese uno de los motivos que llevaran a la salida de Venezuela del bloque en 2006. Contemporáneamente, es posible afirmar que uno de los grandes problemas a los que se ha enfrentado el bloque tiene que ver con la adopción de Acuerdos Bilaterales que involucran a países miembros del bloque, sobre todo Perú y Colombia, y las grandes potencias occidentales (EEUU y Unión Europea), lo cual viene generando un desestimulo al comprometimiento de tales países con el desarrollo del proceso de integración andino.

Es cierto que la integración andina no se encuentra exenta de críticas, sobre todo, por su capacidad de actuar efectivamente en el desarrollo de la región. Blanco AlvarAdo señala que la institucionalización de la Comunidad Andina no produce los efectos deseados por fuerza de factores políticos y jurídicos que preceden los de orden económico y no permiten el avance del proceso de integración, y la falta de preparación de los países miembros para comportarse en un ambiente de integración. Interesante apuntar que entre tales factores políticos y jurídicos es posible señalar la ausencia de legitimización del proceso de integración frente al pueblo andino «comoquiera que el mismo no siente que dicho proceso contribuya con soluciones a sus problemas socioeconómicos», estando clara la distancia entre Comunidad Andina y sociedad civil ${ }^{25}$.

Tal hecho demuestra la necesidad de mayor cohesión entre Órganos e Instituciones que componen la Comunidad Andina y el Sistema Andino de Integración, preparando los países miembros para asumir sus responsabilidades ante la integración y conferir mayor participación política a los ciudadanos en ese proceso. El alejamiento de la sociedad civil deja la conducción de la integración andina apenas en nivel institucional disminuyendo su magnitud y relevancia en lo que se refiere a un factor fundamental: la integración de los pueblos andinos.

A pesar de las discordancias internas entre sus gobernantes, se verifica un consenso en relación a la necesidad de mantener y fortalecer la cooperación entre los países miembros de Comunidad Andina y desarrollar su proceso de integración como forma de introducir y conferir a la región un papel relevante en el escenario político y comercial internacional y hacer frente a los desafíos impuestos por el la era de la globalización.

Como punto final, podemos constatar que la Comunidad Andina surge como un proceso de integración que va más allá de la creación de una simple zona de negociones comerciales interregionales. Su objetivo es

${ }^{25}$ Blanco Alvarado, C. La Crisis de la Comunidad Andina. Revista de Derecho, Universidad del Norte, 42: 1-32, Barranquilla, 2014. 
alcanzar un espacio decisiones comunes sobre política, economía y sociedad, ambición que tiene sus primeros señales de avances en la década de 90, cuando se desarrolla la Unión Aduanera y la propuesta de establecimiento de un Mercado Común en la región, además de documentos que manifiestan la idea de los países miembros de trabajar en conjunto en la superación de retos de contenido político y social. No obstante, conforme señala CASAS GRAGEA, todos estos planteamientos y avances surgen de la retórica política de los principales dirigentes andinos, aunque en realidad los países de la región están inmersos una aguda crisis económica y política que coloca en riesgo el futuro la superación de las etapas necesarias para la consolidación de la integración económica, política y social en la región ${ }^{26}$.

\section{CONCLUSIONES}

La integración regional en Latinoamérica y sus consecuentes beneficios siempre fueron comprendidos como un reto a ser logrado por los países de la región, que en sus relaciones intergubernamentales siempre privilegiaran sus intereses nacionales en perjuicio de una política comunitaria que posibilite el desarrollo conjunto en el continente. Es cierto que en los procesos de integración es necesaria una preparación previa por parte de las Naciones pactantes para no solo disfrutar de sus ventajas sino también hacer cargo de los objetivos que deben ser concretados para proporcionar la existencia harmónica del bloque, desafío que puede ser verificado en el desarrollo histórico del regionalismo andino.

Con relación a la temática institucional, uno de los grandes problemas de la consolidación del proceso de integración andina es la utilización de la Directrices del Consejo Presidencial Andino en asuntos estratégicos para la evolución del bloque justificado por la agilidad y flexibilidad de tales instrumentos. Conforme señalamos anteriormente, las mencionadas Directrices no componen el orden jurídico comunitario, no habiendo implicaciones jurídico-institucionales por no cumplimiento. Además, esa tendencia revela el aspecto intergubernamental que domina la dirección política de la Comunidad, la defensa de los intereses nacionales en detrimento de los comunitarios, lo

${ }^{26}$ En las propias palabras del mencionado autor: «aunque no se puede negar el incremento en los flujos comerciales entre los países miembros, la Unión Aduanera está teniendo serios problemas para consolidarse, y la mayoría de los países de la subregión están viviendo una aguda crisis económica y política. Todo esto no augura un porvenir fácil ni para la consolidación de las etapas de integración económica ni para la puesta en marcha de la integración política y social» (CASAS GrageA, Ángel. La Comunidad Andina: 30 años en Busca del Desarrollo. Revista de Fomento Social 56, p. 65/91, 2001). 
que lleva al cuestionamiento sobre la necesidad de determinados Órganos, debilitando aún más la evolución del proceso de integración.

Por lo tanto, constatamos en tal contexto que determinados Órganos necesitan de una reformulación para mejor encaje en los retos propuestos por el bloque, como por ejemplo, el Parlamento Andino, que debería funcionar como entidad dotada de competencias de contenido normativo para actuar como instancia más activa de representación política ciudadana ante el proceso de integración, superando el problema del alejamiento entre pueblo y Comunidad Andina y reforzando la legitimación democrática del ordenamiento jurídico comunitario, lo que le atribuiría un carácter supranacional.

La Comunidad Andina también podría asumir su connotación intergubernamental, estableciendo una nueva arquitectura institucional que redujera la vinculación del sistema jurídico comunitario, determinando nuevos objetivos para la entidad sobretodo en temas políticos y sociales y flexibilizando la participación de los países miembros en el proceso de integración a actuaciones de carácter puntual. Sin embargo, esta situación colocaría en riesgo todas las conquistas obtenidas históricamente por el bloque y quizás su propia existencia, en la medida en que la cooperación en la región estaría nítidamente debilitada. Ante tales afirmaciones concluimos que algo es esencial para el futuro de la Comunidad Andina: definir que camino seguir como entidad supranacional o intergubernamental, para que la cooperación e integración entre los países de la región pueda continuar existiendo.

\section{REFERENCIAS BIBLIOGRÁFICAS}

Blanco Alvarado, C., La Crisis de la Comunidad Andina. Revista de Derecho, Universidad del Norte, 42: 1-32, Barranquilla, 2014.

Casas Casas, A. y Correa, M. E., ¿Qué Pasa con la Comunidad Andina de Naciones-CAN? Papel Político vol. 12, n. ${ }^{\circ}$ 02. Bogotá: Pontificia Universidad Javeriana, 2007.

Casas Gragea, Ángel María, El Modelo Regional Andino: Enfoque de Economía Política Internacional. Quito: Universidad Andina Simón Bolívar, 2003.

—_La Comunidad Andina: 30 años en Busca del Desarrollo. Revista de Fomento Social 56, p. 65/91, 2001.

Contipelli, E. y Picciau, S., La Evolución de los Modelos de Cooperación Internacional y las Potencias Emergentes: BRICS. Sao Paulo: Revista Direito Mackenzie v. 08, 2014.

DuRAn Valdés, L., El Acuerdo de Integración Subregional: Antecedentes, Negociaciones y Comentarios. Revista de Derecho Publico n. ${ }^{\circ}$ 10. Santiago: Universidad de Chile, 1969.

García-Guerrero, J. L., Las Integraciones Económicas Supraestatales y los Acuerdos entre Bloques Económicos en Definitiva, la Globalización como Último embate al Concepto Racional Normativo de Constitución, en PraTs, E. J. y JIMI- 
NIÁN, M. V. (coords) Constitución, Justicia Constitucional y Derecho Procesal Constitucional. Republica Dominicana: Instituto Dominicano de Derecho Constitucional, 2014.

Germánico, S. P., El Grupo Andino de Hoy: Eslabón Hacia la Integración de Sudamérica. Quito: Universidad Andina Simón Bolívar, 1995.

GonzÁles, A. C., Reflexiones sobre el Futuro de la ALALC. Integración Latinoamericana: El Futuro de la ALALC. Revista Mensual INTAL n. ${ }^{\circ}$ 40, 1979.

Leví Coral, M., La Relación UE-Comunidad Andina en el Contexto de la Negociación de los Acuerdos de Asociación en Roy, J. y Domínguez, R. (eds). España en la Unión Europea y el Estado de la Integración Latinoamericana. Miami: Miami-Florida European Union Center/Jean Monnet Chair, 2010.

Luna CAMPo, A., Elementos de Derecho Comunitario: Panorámica de la ALALC y del Pacto Andino. Bogotá: PAX, 1974.

MAZZEI, Franco, Relazioni Internazionali. Milano: Egea, 2012.

QuINDIMIL, J., Instituciones y Derecho de la Comunidad Andina. Bogotá: Tirant lo Blanch, 2006.

Pino Estrada, M. M., El Sistema Jurídico en la Comunidad Andina de Naciones. Revista Intellector n. ${ }^{\circ}$ 04, Rio de Janeiro: CEGRI, 2006.

Porta, F., La Integración Sudamericana en Perspectiva. Problemas y Dilemas. Santiago: CEPAL, 2008.

Romero, M., Comentario Internacional: Integración Andina en el Contexto de la Crisis Subregional. Revista del Centro Andino de Estudios Internacionales. Quito: Universidad Andina Simón Bolívar, 2001.

TANGarife, M., Derecho de la Integración en la Comunidad Andina. Bogotá: Baker \& McKenzie, 2002.

TITLE: The Andean Community of Nations and the evolution of the process of economic integration in Latin America

RESUMEN: El presente artículo tiene por objetivo realizar una descripción general del proceso de integración andino a partir de su análisis histórico, institucional y conceptual. De ese modo, en la primera parte, presentamos un breve histórico del desarrollo institucional de la integración andina, verificando los principales hechos que contribuirán para su formación y que actúan en su conformación actual. La segunda parte explora la estructura institucional de la Comunidad Andina sobre todo para verificar la adecuación entre sus órganos e instituciones con los objetivos estipulados para el bloque. En la tercera parte, tratamos de la categorización del regionalismo andino y la evolución de sus propuestas institucionales, especialmente, para verificar como la Comunidad Andina se comporta ante el actual escenario globalizado, como forma de debatir el propio cuadro del desarrollo del proceso de integración socioeconómica en Latinoamérica.

Palabras Clave: Comunidad Andina. Integración. Regionalismo. América Latina. 
ABSTRACT: The present article aims to realize a general description of the Andean integration process from its historical and conceptual analysis. Therefore, in the first part, we present a brief historical of the institutional development of the Andean Integration, checking the principals facts that contribute to its formation and that act in its current conformation. The second part explores the institutional structure of the Andean Community specially to verify the compatibility among its entities and institutions with the objectives proposes for the block. In the third part, we investigate de categorization of the Andean regionalism and the evolution of its institutional proposes, specially, to verify how the Andean Community act in the globalized scenario as a way to debates the framework of the development of the socioeconomic integration process in Latin America.

KEY WORDS: Andean Community. Integration. Regionalism. Latin America.

RECIBIDO: 03.06.2016

ACEPTADO: 22.06.2016 